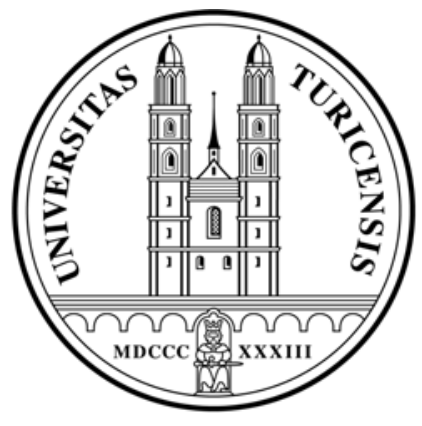

Institute for Empirical Research in Economics

University of Zurich

Working Paper Series

ISSN 1424-0459

Working Paper No. 347

\title{
Deadlines and Distractions
}

Maria Saez-Marti and Anna Sjögren

December 2007 


\title{
Deadlines and Distractions.*
}

\author{
Maria Saez-Marti \\ University of Zürich
}

\author{
Anna Sjögren \\ IFN, Stockholm
}

December 13, 2007

\begin{abstract}
We consider a principal-agent model in which a task, demanding a sequence of efforts by the agent, must be completed by a certain date. Effort is not contractible. Agents are subject to shocks affecting their opportunity cost of time such that they are distracted from work when the opportunity cost of time is high. We show that the probability that a task is completed by the deadline is a non-monotonic function of the agent's probability of being distracted. The anticipation of future distractions induces rational agents to get started earlier for precautionary reasons. As a result, agents who are more often distracted may outperform agents who are distracted less often. Principals can increase the probability that the task is completed, and thus achieve higher profits, by strategically setting "tight" deadlines, provided that these can later be extended with a positive probability.
\end{abstract}

Keywords: Deadlines, time-consistency, timing of effort, optimal incentives.

JEL-codes D81, J22, M50.

${ }^{*}$ We thank Dirk Niepelt, Philippe Sulger, Fabrizio Zilibotti and participants at the EEA 2004 meetings for helpful comments. Financial support from Vetenskapsrådet is gratefully acknowledged. 


\section{Introduction}

Many situations are characterized by deadlines. Deadlines are usually associated with tasks that take time to complete and such that failing to meet the deadline implies that all or a substantial fraction of the effort exerted up to that point is wasted. In principle, deadlines are supposed to discipline the timing of agents' effort. However, for those of us who sometimes get distracted from working, the risk of wasting effort may make deadlines discouraging. In fact, this is why we may fail to get started reading a long paper on a Friday afternoon. The risk is overwhelming that we only get half way through because a colleague asks us to come for a drink.

Distractions, whether in the form of a more acutely pressing task at work or a sick child requiring a parent to stay at home from work, put the completion of the task at risk. We may respond in different ways to this risk. One possibility is that the risk of being distracted is so large that we do not find it worthwhile to get started on the task at all. Another possibility is that the presence of distractions actually helps and encourages us to get started reading that paper right away on Friday morning. We know we had better work early, in case we get distracted later, if we are ever to get through before we call the day.

Rational agents who discount future rewards and costs would tend to work as late as possible, to finish just on time. However, when they are aware that they may get distracted along the way, they trade off their aversion to work early with the desire to avoid failing to meet the deadline because of later distractions.

There are numerous examples involving such trade off. Grant or job applications, submission of term and conference papers, reaching of sales targets to get bonus payments or a contract to deliver a good or a service at some specific date are but a few. We can also think of tenure decisions and probation periods in employment contracts as examples of such deadlines. In all these examples, the task requires repeated efforts over time and there is a risk of being only half way through when the deadline expires.

There is evidence that distractions and the time consuming nature of some tasks are important determinants of when people get started. Being distracted by too many other things to do is the most frequently stated reason for academic procrastination and failure to meet deadlines according to Solomon and Ruthblum (1984). Moreover, Boice (1989) documents that it is because some tasks, e.g. academic writing, are perceived as requiring long periods of work that researchers view themselves too busy to get their writing going.

The first objective of this paper is to analyze how occasional distractions affect the timing of work on tasks that require the agents full attention for more than one period in the presence of deadlines.

We find a non-monotonic relation between distractions and the probability that tasks are completed. In some cases agents who are subject to more frequent distractions are actually more likely to finish the job than agents who are less easily distracted. Of course this is not 
always true. Agents who are never distracted, always carry out the task at the last moment and always complete. Agents who are distracted most of the time never even get started. The interesting result arises for moderate likelihoods of being distracted when the risk of being distracted induces precautionary effort. By getting started early, agents who are distracted more frequently complete the task more often than agents who are less frequently distracted and therefore do not exert any precautionary effort.

There is ample evidence in the real world of deadlines that get extended, or simply broken. ${ }^{1}$ Some deadline contracts even specify explicit conditions for the possible extensions. Apart from examples concerning compliance with regulations, and delivery of commentaries or advice, there are uncountable announcements of extended deadlines for submission of papers to academic congresses or submissions of candidates for various prizes or for submitting tenders. $^{2}$

This observation brings us to the second result of the paper. We show that principals dealing with rational agents who are subject to preference shocks, or distractions that increase the opportunity cost of their time, may find it optimal to set stochastic deadlines. In particular, suppose that a task must be completed by time $T$. The principal may increase the probability that the job is done by setting a deadline which is one period shorter, while retaining the option to extend it for one more period with positive (but less than unit) probability. We refer to such shorter deadlines as a "tight" deadlines. Tight deadlines are valuable since they encourage the agent to start earlier for precautionary reasons. However, stochastic extensions are also useful as they reduce the probability that projects are first started and then abandoned due to a sequence of distractions. Our result requires that principals be able to commit to stochastic termination. If the principal could renegotiate the contract ex-post, he would always grant, the extension, and no tight deadline will ever be credible. However, in many instances, the circumstances or procedures may partially tie the principal's hands. It may, for instance, be difficult, though not impossible, to call for an extraordinary board meeting. Our paper shows that this type of uncertainty due to imperfect commitment on the part of the principal may be beneficial and lead to better outcomes than both full commitments and total lack of it.

Our theory can explain in a rational choice framework why deadlines are first imposed, but then often extended, postponed or broken and why in some instances, agents are not granted maximal time at the outset. Our explanation contrasts with that in O'Donoghue and Rabin (1999) who argue that tight deadlines can only be optimal if agents have time-inconsistent

\footnotetext{
${ }^{1}$ For example, in April 2003, the Europeean Commission granted an extension of deadline to the CESR (Committee of European Securities Regulators) to deliver technical advice on implementing measures for a directive, and in December 2001, the US Congress extended the deadline to comply with the Health Insurance Portability and Accountability Act (HIPAA) Transactions and Code Sets requirements. See also Toxvaerd (2006) for a review of evidence on more or less systematic time over runs in a number of industries.

${ }^{2}$ In fact, a google-search for "Extension of the deadline" gave some 182000 hits in January 2007.
} 
preferences. In their framework, tight deadlines can help agents overcome their self-control problems. However, they claim that if agents are time-consistent granting maximal time at the outset is optimal. Counter to this claim, our results show that this is not the case when agents are sometimes distracted.

Our paper is also related to Fischer (2001) and Toxvaerd (2006; 2007). Fischer models time as an exhaustible resource and studies the optimal allocation between effort and leisure when agents face a deadline. Her focus is not on distractions but on the effect of impatience on procrastination. She shows that high rates of time preference lead to dynamically consistent procrastination which manifests itself in increasing workloads. When the task involves more than one stage, and there is uncertainty concerning the workload needed to finish the different stages, more impatient agents may optimally decide to give up. Toxvaerd (2006) analyses project delays and optimal contracts under moral hazard in a setting with time to build and discounting. In his model, the agent must complete a number of sub-projects in a prespecified order. The success in each sub-project depends on the effort exerted. Inefficient delays can be ameliorated by long-term contracts under which the agent is rewarded along the way. Current wages and future rewards based on success reinforce each other in providing incentives to the agent. Toxvaerd (2007) analyses the optimal deadline contract when there is time to build and adverse selection because agents differ in efficiency. Deadlines are used as a screening device and the optimal contract features inefficient delays. As in Fischer, the agent's effort is strictly increasing over time, which we shall see is not necessarily the case in this paper. Instead, we find that the agent may find it optimal to get started on a task and then take a brake, and postpone the completion of the project.

The paper proceeds as follows. In section 2 we introduce a simple decision theoretic model of an agent working against a deadline. We analyze how timing of effort and probability of completion depend on the likelihood of being distracted and on the length of the deadline. In section 3, we embed the model in a principal-agent framework, letting the deadline regime be chosen by a principal. We discuss the conditions under which setting and extending or postponing deadlines is in the interest of the principal. Section 4 concludes.

\section{The model}

We construct a discrete-time model where a principal has a project that must be completed within a deadline. The principal must delegate the project to an agent with specialized skills, and this raises a standard agency problem. The project takes time to complete. More precisely, the agent must spend two full time periods on the project, although the two periods need not be consecutive. In every period, the agent's opportunity cost is high, $\bar{u}$, with probability $p$ and low, $\underline{u}$, with probability $(1-p)$. When the opportunity cost is high, the agent is "distracted" from work. The source of fluctuation could be either that agents are occasionally offered other 
more lucrative projects or that agents are unable to work on the project because of illness or other reasons preventing them from working.

Any effort by the agent has a relation-specific value with no return outside the specific principal-agent relation. A completed venture, however, generates a total value $W>0$ which can be realized at time $T$. A project completed ahead of the deadline can be stored at zero cost until its value is realized at time $T$. The contractual environment is characterized by severe imperfections. First, we assume that the value $W$ is split according to ex-post bargaining at $T$ resulting in the payoff $B$ to the agent and $(W-B)$ to the principal. As a result, $B$ is taken as given. Second, the costs associated with monitoring and verifying the agent's progress are prohibitively high, so the principal can only commit to rewarding the agent $B$ at $T$ for a task completed within a stipulated deadline $T$. We relax these assumption in section 3.2.

\subsection{The agent's decision}

For an agent to meet the deadline at $T$ and obtain the reward $B$, the last opportunity to complete the project is on the eve of $T$, i.e. at $t=T-1$. In each period $t=0,1,2, \ldots, T-1$, the agent decides whether to work or not after observing the realization of his opportunity cost. The decision to work depends on whether the agent has worked before or not, on the realization of the opportunity cost $u_{t} \in\{\underline{u}, \bar{u}\}$ and on the number of periods left before the deadline expires $(T-t)$. We assume the agent to be rational and to maximize the present discounted expected utility. We assume that the agent discounts the future exponentially, i.e. he is time consistent.

We start first with the decision to complete an already started project. Note that the decision to complete a task is equivalent to the decision to undertake a project that requires only one period of effort.

\section{The decision to complete.}

Consider first an agent who has already worked one period and arrives at the eve of the deadline without having completed. He will complete the project if the discounted payoff to meeting the deadline outweighs the opportunity cost of working, i.e., if

$$
\delta B \geq u_{T-1}
$$

where $u_{T-1} \in\{\underline{u}, \bar{u}\}$, and $\delta \in(0,1]$ is the agent's discount factor.

Since the focus of the analysis is on the agent's timing of effort, and on the probability of completing the task when there is competition for his time or attention, we assume that this competition is fierce in one particular respect: The agent never works on the deadlined task when the opportunity cost of time is high. However, the project is worth completing when he is not distracted. Throughout the paper we therefore maintain the following assumption:

Assumption 1: $\delta B \in[\underline{u}, \bar{u})$. 
Under this assumption the agent never works when $u_{t}=\bar{u}$. Given that a distracted agent never works, we can focus on the determinants of the agent's decision to work or not conditional on the opportunity cost being low.

Consider an agent who is distracted with probability $p$ and let $c_{T-t}(p)$ denote the value of the project that makes him indifferent between completing at $t$ and postponing completion. The subscript $T-t$ indicates the number of remaining periods before the deadline expires. Provided that the opportunity cost is low, the agent completes the project $k$ periods ahead of the deadline only if $B \geq c_{k}(p)$. Whenever $B<c_{k}(p)$ the agent postpones completion. The condition, $c_{k}(p)$, depends on the agents willingness to complete in later periods i.e. on $c_{\tau}(p)$, $\tau<k$. The following proposition characterizes the complete conditions $c_{k}(p), k=1,2, \ldots$

Proposition 1 The agent is willing to complete $k$ periods ahead of the deadline if $B \geq c_{k}(p)$, where

$$
c_{k}(p)=\frac{\underline{u}}{\delta^{k} p^{k-1}}\left(1-(1-p) \frac{\delta p-(\delta p)^{k}}{p(1-\delta p)}\right),
$$

i) $c_{k+1}(p)>c_{k}(p)$ for all $k=1,2, \ldots$

ii) $c_{1}(p)=\underline{u} / \delta$, and for $k \geq 2, \lim _{p \rightarrow 0} c_{k}(p)=\infty, c_{k}^{\prime}(p)<0$, and $c_{k}(1)=\underline{u} / \delta^{k}$.

Proof. See appendix

Part i) of Proposition 1 establishes that an agent who is willing to complete a task $k$ periods ahead of the deadline, is also willing to complete the task as the deadline approaches. Part ii) establishes that an agent who is never distracted $(p=0)$ always postpones completion until the eve of the deadline and that the more distracted the agent (higher $p$ ), the lower the payment needed to induce early completion. Proposition 1 also implies that, given $p$ and $B \geq \underline{u} / \delta$, there is a critical threshold $k^{c}(p, B) \geq 1$, such that the agent prefers to postpone completion if there remain more than $k^{c}(p, B)$ periods before the deadline expires, but completes at the first realization of low opportunity cost if there are $k^{c}(p, B)$ or fewer periods left, i.e. when $t \geq T-k^{c}(p, B)$. Figure 1 illustrates the optimal decision to complete.

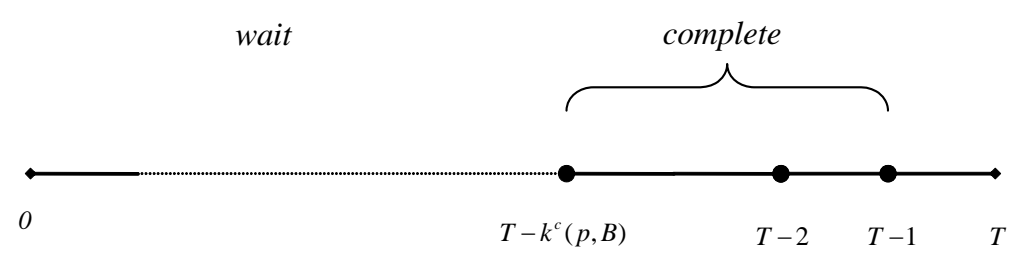

Figure 1: The decision to complete

\section{The decision to start.}

We proceed to analyze the decision to get started on a project that requires two periods of effort. This decision depends on both future start and future complete conditions. We first illustrate the derivation for $k=T-2$, and then obtain a general expression. 
The last period the agent may consider to start is $T-2$. Under Assumption 1, the agent knows that if he starts at $T-2$ he completes at $T-1$, provided that he does not get distracted. He therefore starts at $t=T-2$ if the opportunity cost of time is low and

$$
\delta^{2}(1-p) B+\delta p \bar{u} \geq \underline{u}+\delta(p \bar{u}+(1-p) \underline{u}) .
$$

Note that, because the agent never works when the opportunity cost of time is high, all conditions to work depend only on $p, \delta, B$ and $\underline{u}$, while terms in $\bar{u}$ cancel out. The inequality in (2) can be rewritten as the following start condition:

$$
B \geq \frac{\underline{u}}{\delta^{2}} \frac{1+(1-p) \delta}{(1-p)}=s_{2}(p)
$$

i.e. the reward required to make the agent to start when the deadline is two periods away. It is easy to see that $s_{2}(p)$ is increasing in $p$, moreover, $s_{2}(p)>\underline{u} / \delta$ for all $p$ and $\lim _{p \rightarrow 1} s_{2}(p)=\infty$. This implies that the higher the likelihood of being distracted, the higher is the reward needed to induce the agent to start working in the project. Since $s_{2}(p)>\underline{u} / \delta$, the agent is only willing to start at $T-2$ if he is certain to complete at $T-1$, given a low opportunity cost. We can now obtain the probability of meeting a deadline which is two periods away, $\pi_{2}(p, B)$. The project is undertaken and completed only if the agent has two consecutive periods of low opportunity cost of time and the payoff, $B$, is high enough, namely

$$
\pi_{2}(p, B)=\left\{\begin{array}{l}
0 \text { if } B<s_{2}(p) \\
(1-p)^{2} \text { if } B \geq s_{2}(p) .
\end{array}\right.
$$

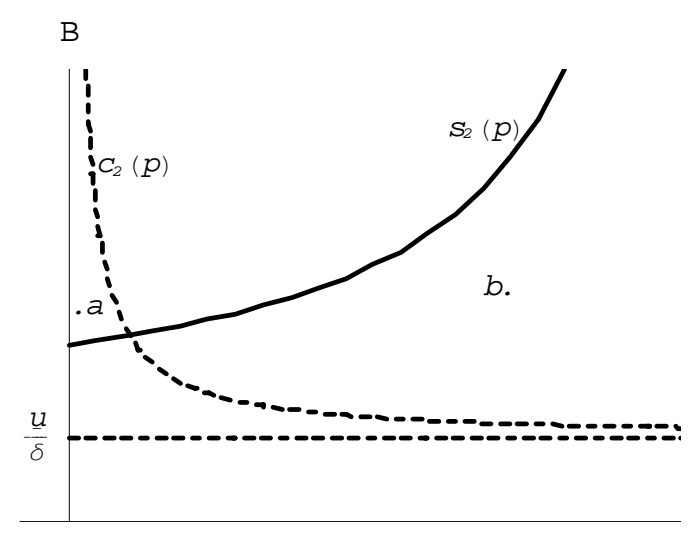

Figure 2: Start and complete conditions for $k=2$.

Figure 2 illustrates the start and complete conditions when the deadline is two periods away, $s_{2}(p)$ and $c_{2}(p)$, and $c_{1}(p)=\underline{u} / \delta$. In periods of low opportunity cost, the agent only starts (completes) two periods ahead of the deadline when the pairs $(p, B)$ lie on or above 
$s_{2}(p)\left(c_{2}(p)\right)$. For instance for $(p, B)=a$, the agent starts the project, but takes a break (i.e. postpones completion) if the project is already under way. Instead, for $(p, B)=b$, the agent is not willing to start, but completes early if the project is already under way. Note that the more easily distracted the agent, the more willing is the agent to complete the task ahead of the deadline, but the less willing is the agent to get started.

Now, consider the decision to start $k$ periods ahead of the deadline. Let $s_{T-t}(p)$ denote the start condition at time $t$ when the deadline is at $T$. As before, the subscript $T-t$ indicates the number of periods remaining before the deadline expires. The start condition $s_{k}(p)$ gives the reward that makes the agent indifferent between starting at $T-k$ and not starting. It depends on both future complete and future start conditions, since the agent must compare the consequences of getting started immediately with the consequences of postponing start, taking into account future behavior.

The following lemma and its corollary establishes important intermediate results that are useful for characterizing the general start condition.

Lemma 1 If the agent is willing to start $k+1$ and $k-1$ periods ahead of the deadline then, he also wants to start $k$ periods ahead, i.e., $s_{k}(p) \leq \max \left\{s_{k-1}(p), s_{k+1}(p)\right\}$ for all $p$ and $k$.

Proof. See appendix

Corollary For all $k=2,3 \ldots$

i) if $s_{k+1}(p) \geq s_{k}(p)$ then $s_{k+2}(p)>s_{k+1}(p)$, and

ii) if $s_{k+2}(p) \leq s_{k+1}(p)$ then $s_{k+1}(p)<s_{k}(p)$.

Lemma 1 implies that, conditional on ever starting, i.e. $B \geq s_{k}(p)$ for some $k$, the set of subsequent periods in which the agent is willing to start is either empty, i.e. $s_{\tau}(p)>B$ for all $\tau<k$, or an interval, i.e. $s_{\tau}(p) \leq B$ for $\tau=k-1, ., k-j$ for some $j \in\{1, . ., k-2\}$. It is therefore the case that for all $(p, B)$ such that $B \geq s_{k}(p)$ for some $k$, there are two critical threshold values, $\underline{k}^{s}(p, B)$ and $\bar{k}^{s}(p, B)$ (where $\underline{k}^{s}(p, B)<\bar{k}^{s}(p, B)$ ), such that the agent is willing to get started on the project only if the number of periods ahead of the deadline is between (and including) the thresholds. Figure 3 illustrates the optimal starting decision. If the deadline is too far off, $T>\bar{k}^{s}(p, B)$, the agent postpones start until $\bar{k}^{s}(p, B)$ or fewer periods remain, but if the agent has not started by $T-\underline{k}^{s}(p, B)$, he never will.

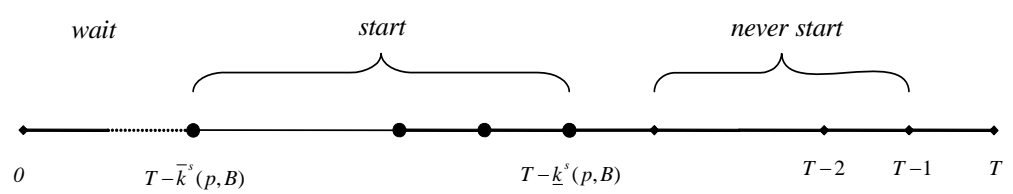

Figure 3: The decision to start

Before we can characterize the general start condition, Lemma 2 establishes a useful relation between the earliest period in which the agent is willing to complete the task $k^{c}(p, B)$, and the last period in which he would want to start, $\underline{k}^{s}(p, B)$. 
Lemma $2 k^{c}(p, B) \geq \underline{k}^{s}(p, B)-1$.

Proof. See appendix.

Lemma 2 implies that if $T-t$ is the last period the agent wants to start, then he wants to complete at the first opportunity. Making use of Lemma 1 and Lemma 2, we now obtain the condition $s_{k}(p)$ that makes the agent indifferent between starting and postponing start when the deadline is $k$ periods away, taking into account that he will behave optimally in the future. The following proposition summarizes the properties of $s_{k}(p)$.

Proposition 2 The agent is willing to start $k$ periods before the deadline if $B \geq s_{k}(p)$, where (i) $s_{2}(p)$ is increasing in $p, s_{2}(0)=\underline{u} / \delta^{2}$, and $\lim _{p \rightarrow 1} s_{2}(p)=\infty$;

(ii) for all $k \geq 3, s_{k}(p)$ is $U$-shaped, $\lim _{p \rightarrow 0} s_{k}(p)=\infty$, and $\lim _{p \rightarrow 1} s_{k}(p)=\infty$; and

(iii) $s_{k}(p)>s_{k-1}(p)$ for all $p$ if $\delta \leq 1 / 2$, otherwise $s_{k}(p)<s_{k-1}(p)$ for large $p$ 's provided $\delta>(k-2) /(k-1)$.

Proof. See appendix.

We illustrate the results in Propositions 1 and 2 in Figure 4, where we have depicted the start conditions with solid lines for $k=2$ (black), 3 (dark gray), 4 (medium gray) and 5 (light gray). We have also plotted the complete conditions for $k=1,2,3,4$ (dashed lines). An agent is willing to start (complete) the task $k$ periods ahead of the deadline for all combinations of parameters $(p, B)$ lying above $s_{k}(p)\left(c_{k}(p)\right)$. Else, he prefers not to start (complete).

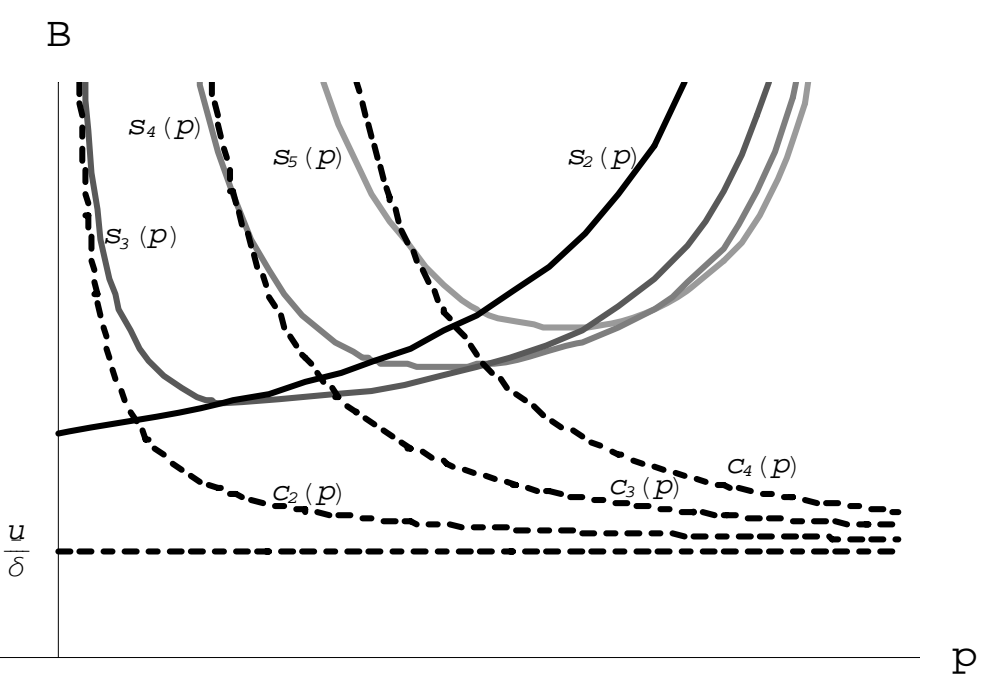

Figure 4. Start conditions for $k=2,3,4,5$ complete conditions for $k=1,2,3,4$.

First, as shown by Proposition 1, the payoff required to complete a task under way is lower the closer the deadline and the more frequently distracted the agent. The reason is that delaying completion jeopardizes the possibility of meeting of the deadline. Second, agents who 
are never distracted always postpone effort until the last period: when the deadline is more than two periods (one period) ahead, the start (complete) condition goes to infinity as $p$ goes to 0 . Third, agents who get distracted most of the time (high $p$ ), never get started. Hence, for a given deadline and a given payoff, the rarely distracted and the often distracted are least willing to get started although for different reasons. The rarely distracted are reluctant to start because due to discounting they prefer to postpone since they are sure to complete anyway. The very distracted are instead reluctant to start because the risk of failing to meet the deadline is so high that they do not find it worthy to sink one period of effort. It is worth noting that two start conditions cross once, if at all.

Figure 4 also illustrates the possibility of breaks. It can be shown that when $k>2+\delta^{-1}>$ 3 , the optimal number of breaks is positive for some values $(p, B)$. In the example depicted in Figure 4 , the start condition $s_{4}(p)\left(s_{5}(p)\right)$, and the complete condition for $c_{3}(p)\left(c_{4}(p)\right)$, cross. For values of $p$ and $B$ below $c_{3}(p)\left(c_{4}(p)\right)$ and above $s_{4}(p)\left(s_{5}(p)\right)$, an agent who gets started at $T-4(T-5)$, will take a break at $T-3(T-4)$ and complete at first opportunity from $T-2$ $(T-3)$ onwards. An intuition for this result is that getting started early is an investment in the probability of completing. Since the deadline is still far ahead, an agent who is only moderately distracted is willing to grant himself a break as long as this does not compromise the probability of completing enough to outweigh the break's implied earlier enjoyment of leisure utility in times of low opportunity cost of time, $\underline{u}$. Agents who are more distracted also get started early, but they do not take breaks.

\subsection{Getting the task done}

We now turn to how the likelihood of being distracted affects the probability that an agent completes a task. We compute the ex-ante probability, at time zero, that agents with different $p$ 's complete a task with a deadline $T$ periods later. The agent starts in the first period if the opportunity cost is low and $B \geq s_{T}(p)$. Then, the probability of completion, depends on whether the agent takes breaks or not. The agent completes without breaks if $B \geq c_{T-1}(p)$ and takes breaks if $B<c_{T-1}(p)$. If the opportunity cost of time happens to be high in the first period or if $B<s_{T}(p)$, then the probability of completing the task is the same as if the deadline had been one period closer. Hence, for $T \geq 3$ we can write the probability of completing the task as:

$$
\pi_{T}^{*}(p, B)=\left\{\begin{array}{cc}
\pi_{T-\bar{k}^{s}(p, B)}^{*}(p, B) & \text { if } T>\bar{k}^{s}(p, B) \\
p \pi_{T-1}^{*}(p, B)+(1-p)\left(1-p^{\min \left\{T-1, k^{c}(p, B)\right\}}\right) & \text { if } T \leq \bar{k}^{s}(p, B)
\end{array},\right.
$$

where $\min \left\{T-1, k^{c}(p, B)\right\}$ is the number of periods in which the agent is willing to complete the task, provided that he started $T$ periods ahead of the deadline, and where $\pi_{2}^{*}(p, B)$, is given by(4). Proposition 3 characterizes how the probability of completion in Equation (5), depends on $B, p$, and $T$. 
Proposition 3 Assume $B \geq s_{k}(p)$ for some $k \leq T$, then

i) $\pi_{T}^{*}(p, B) \geq \pi_{T-1}^{*}(p, B)$ for any $T$;

ii) the probability of completion $\pi_{T}^{*}(p, B)$ is an increasing step function of $B$;

iii) $\pi_{T}^{*}(p, B)$ is piecewise decreasing in $p$, and for $T \geq 3, \pi_{T}^{*}(p, B)$ exhibits a finite number of upward discontinuities, provided $B \geq s_{k}(p)$ for some $p$ and some $k>2$.

Proof. See appendix

Proposition 3 shows that increasing the length of the deadline, or the payoff, to the agent never reduces the probability of completion. Proposition 3 also implies that agents with higher levels of distraction (higher $p$ ) can have higher success rates than agents who are less likely to be distracted, provided that the risk of being distracted makes them start working on the task earlier. This important result is illustrated in Figure 5.

Figure 5 shows, for a given $B$, how the probability of completion, $\pi_{T}^{*}(p, B)$, changes with the length of the deadline, $T$, and with $p$. The thin solid line is $\pi_{2}^{*}(p, B)$, the thin dashed line is $\pi_{3}^{*}(p, B)$ and the thick dashed line is $\pi_{4}^{*}(p, B)$.

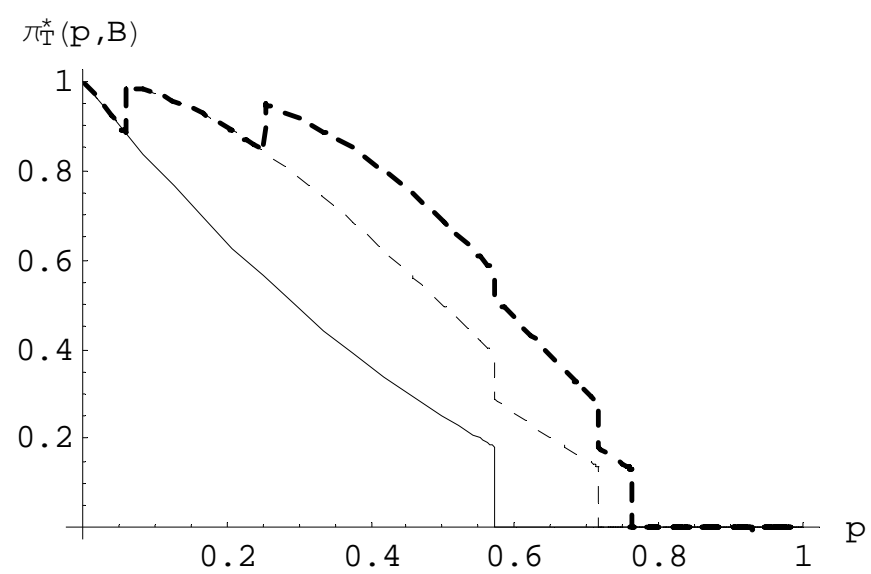

Figure 5: Probability of completion for $T=2,3,4$ as a function of $p$.

Adding time, makes no difference to those who are only rarely distracted ( $p$ close to 0 ). They never start working until two periods before the deadline. Instead, granting longer deadlines matters as $p$ increases, since more distracted agents find it optimal to get started right away. Given $T>2$, the probability of completion exhibits upwards jumps at those $p$ 's that encourage immediate start. Note that for $T=3$, there is only one upward jump at the $p$ which encourages starting three periods before the deadline while for $T=4$, there is second upward jump at the $p$ that encourages start four periods before the deadline. 


\section{The principal's decision}

We now proceed to the principals decision to set a deadline for the venture, taking the behavior of the agent as given. Let $W$ be the joint surplus of the project and assume that it can only be realized at $\hat{T}$. The strategy of the principal consists of a deadline at $T \leq \hat{T}$, given a payment to the agent $B<W$. The present discounted expected profit of the principal, $\Pi(T, B)$, is

$$
\Pi(T, B)=\left(\delta^{\hat{T}} W-\delta^{T} B\right) \pi_{T}^{*}(p, B)
$$

where $\delta^{\hat{T}} W-\delta^{T} B$ is the present value of the principal's surplus and $\pi_{T}^{*}(p, B)$ is the probability that the agent completes the project.

Proposition 3 implies that $\Pi(T, B) \leq \Pi(\hat{T}, B)$ for all $T<\hat{T}$. The probability of completion is non-decreasing in the time horizon, and allowing for maximal time maximizes the principal's payoff. This result is consistent with O'Donoghue and Rabin (1999) who argue that timeconsistent agents should be granted maximal time. However, in our model with "distracted" agents, the result only holds if deadlines are set deterministically. The non-monotonicity in the probability of completion discussed in the previous section, suggests that it would be possible to improve the probability of completion further if the principal could somehow induce an agent to get started earlier. In what follows, we show that if principals can introduce uncertainty about the length of the deadline, they may find it optimal, contrary to O'Donoghue and Rabin (1999), to set an initial deadline tighter than that imposed by nature.

\subsection{Stochastic deadlines}

We introduce the possibility of setting stochastic deadlines. We say that the principal sets a stochastic deadline at $T$ if he initially sets period $T-1$ as the "tight" deadline for the project, but that he when the tight deadline expires extends the deadline to $T$ with some predetermined positive probability $q \in[0,1]$. We refer to the deadline at $T$ as the "natural" deadline, since $T$ is the period in which the value of the project can be realized, and to the deadline at $T-1$ as the "tight" deadline. We assume that the uncertainty about the extension is resolved at $T-1$, so that tasks under way may not be completed if the true deadline turns out to be tight. As in previous sections, we assume that the value of the project, $W$, can only be realized at $T$, and that $B$ is the resulting payment to the agent if the two parties were to bargain over the surplus at $T .{ }^{3}$ In what follows, we characterize the agent's conditions for starting and completing the task under a stochastic deadline.

\section{The decision to complete.}

\footnotetext{
${ }^{3}$ The same qualitative results follow if we assume that the principal anticipates the payment to the agent. However, the chosen specification is analytically simpler, and allows us to focus only on the effect of the uncertainty on the probability of success, abstracting from additional incentives due to earlier payments.
} 
Let $c_{T-t}^{S}(p, q)$ denote the complete condition at $t$, when the deadline is stochastic at $T$ and the probability of extension of the deadline is $q \in[0,1]$. Assume first that $t=T-1$ and that the deadline is extended, then the relevant conditions for completing is the one obtained in the deterministic case when the deadline is one period away. The agent only considers working if he has worked once, the opportunity cost is low and $\delta B \geq \underline{u}$ (assumption 1). In the appendix we derive a general condition for $c_{T-t}^{S}(p, q)$. The results are summarized in the following proposition.

Proposition 4 The agent is willing to complete the task $T-k$ periods before the stochastic deadline at $T$ when

$$
B \geq \frac{p c_{k}(p)}{1-(1-p) q}+\frac{(1-q)(1-p) \underline{u}}{\delta(1-(1-p) q)}=c_{k}^{S}(p, q), \text { where }
$$

i) $c_{k}^{S}(p, 1)=c_{k}(p), c_{k}^{S}(p, 0)=c_{k-1}(p) / \delta$, and

ii) $\partial c_{k}^{S}(p, q) / \partial q>0, c_{k+1}^{S}(p, q)>c_{k}^{S}(p, q)$ for all $p$.

Proof. See Appendix

Proposition 4 shows that a stochastic deadline at $T$ makes the agent more willing to complete the task relative to the deterministic deadline at $T$. As was the case under a deterministic deadline, the agent willing to complete at a certain period is also willing to complete in all subsequent periods.

\section{The decision to start.}

Let $s_{T-t}^{S}(p, q)$ denote the start conditions at $t$, when the deadline is stochastic at $T$. First, consider the decision to start at at $T-2$. The agent knows that if he starts at $T-2$ he has a chance to complete the task only if the deadline is extended, and the opportunity cost is low. The agent will thus decide to start at $T-2$ when

$$
(1-p) q \delta^{2} B+(1-p)(1-q) \delta \underline{u} \geq \underline{u}+\delta(1-p) \underline{u},
$$

which can be re-written as

$$
B \geq \frac{\underline{u}}{\delta^{2}} \frac{1+(1-p) q \delta}{(1-p) q}=s_{2}^{S}(p, q)
$$

Proposition 4 and (7) imply that the threat of a tight deadline makes the agent less willing to start at $T-2$, but increases the agent's willingness to complete, much in the same way as an increase in the probability of distraction. Now, it is straight forward to compute the probability of completing a task when the deadline is stochastic at 2 :

$$
\pi_{2}^{S}(p, q, B)=\left\{\begin{array}{l}
0 \text { if } B<s_{2}^{S}(p, q) \\
q(1-p)^{2} \text { if } B \geq s_{2}^{S}(p, q) .
\end{array}\right.
$$


A stochastic deadline two periods away, clearly, gives a lower probability of completion than a deterministic deadline of the same length. However, as we move further ahead of the deadline, by proposition 4 , the effect of the stochastic deadline is to make the agent more willing to complete ahead of the deadline compared to a deterministic deadline. This increased willingness to complete has implications for the decision to start. In the appendix we derive the general start condition $s_{T-t}^{S}(p, q)$ when the deadline is stochastic at $T$. Lemma 3 shows that the effect on the decision to start, $s_{k}$, of introducing a stochastic deadline depends only on the relation between the last period the agent wants to start and the first period after $k$ in which he wants to finish.

Lemma $3 A$ stochastic deadline makes the agent more willing to start $k$ periods before the deadline, relative to the deterministic deadline, if

$$
p<\frac{m_{k}(p)-n_{k}(p)+1}{m_{k}(p)-n_{k}(p)+2} \in[0,1),
$$

where $m_{k}(p)=\min \left\{k-1, k^{c}\left(p, s_{k}(p)\right)\right\}$ and $n_{k}(p)=\underline{k}^{s}\left(p, s_{k}(p)\right)$. Otherwise the agent is more willing to start with the deterministic deadline.

Proof. See appendix

Lemma 3 allows us to formulate the following proposition regarding the effect of stochastic deadlines on the decision to get started.

Proposition 5 For $k \geq 3$, the introduction of stochastic deadlines encourages start for small $p$ 's and discourages start for large $p$ 's.

Proof. See Appendix.

The findings for behavior under stochastic deadlines are illustrated in Figure 6 . The U-shaped lines in the figure are the start conditions. The black curve corresponds to the deterministic deadline at $4, s_{4}(p)$, and the gray line to a stochastic deadline at 4 with probability of extension $q=0.5, c_{4}^{S}(p, 0.5)$. The decreasing functions are the complete conditions for $k=3$. The dashed black line corresponds to the deterministic deadline, $c_{3}(p)$, and the dashed gray line to the stochastic deadline, $c_{3}^{S}(p, 0.5)$. Note that the agent is more (less) willing to start for small (large) $p$ 's with the stochastic deadline and more willing to complete for any $p$. The reason is that for low levels of distraction, the threat of a tight deadline will, in fact, make the agent more willing to get started early for precautionary motives. However, for high levels of distraction, chances of completing are already low and the threat of a tight deadline makes completion even less likely. As a result, the agent is discouraged from starting altogether. 


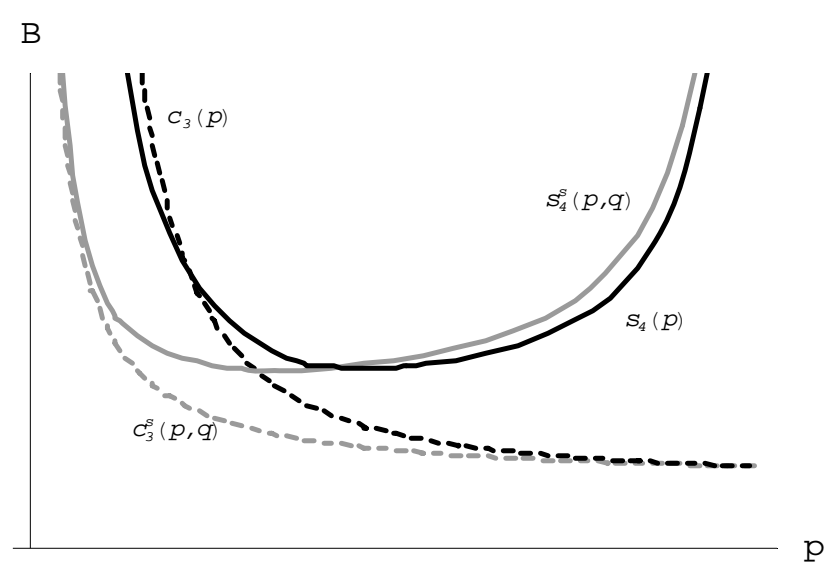

Figure 6: Start $(k=4)$ and complete conditions $(k=3)$ with stochastic and deterministic deadlines.

\subsection{Maximizing profits}

It is clear from Equation (6) that the principal's profits, for a given payment to the agent, $B$, increase with the probability of completion. It is also clear from Proposition 3 that increases in the payoff to the agent, while costly to the principal, do not increase the probability of completion unless the increase is large enough to affect the agent's work behavior. The above analysis, however, shows that a stochastic deadline can improve the probability of completion by inducing the agent to start early with no need of increasing payment $B$. When the principal can set $q$, his profits are

$$
\Pi(T, q)=\delta^{T}(W-B) \pi_{T}^{S}(p, q, B),
$$

where $\pi_{T}^{S}(p, q, B)$ is the probability of completion under a stochastic deadline at $T$.

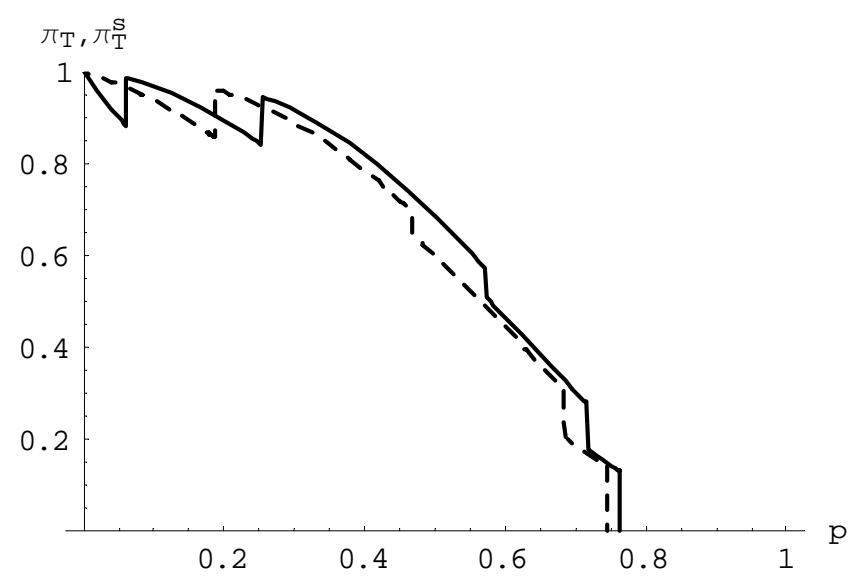

Figure 7: Probability of completion with stochastic and deterministic deadlines $(\mathrm{T}=4)$. 
Figure 7 shows the probabilities of completion under a deterministic deadline at 4 (solid line) and a stochastic deadline at 4 (dashed line). The stochastic deadline results in a higher probability of completion for small and intermediate values of $p$. This is the range of the probability of distraction for which the agent is willing to start one period earlier with the stochastic deadline. In particular the agent starts at $T-3$ (in addition to only at $T-2$ ) when $p$ is small and at $T-4$ (in addition to only at $T-3$ ) for intermediate $p$ 's. Note that when the stochastic deadline does not induce earlier start, the "natural" deadline is better than the stochastic deadline.

This observation is formalized in Proposition 6, which states that a stochastic deadline may be better than the "natural" deadline, provided that the former induces earlier start. Although the stochastic deadline is, by construction, a convex combination of two deterministic regimes, its outcome is not. The reason is that the stochastic deadline for a range of $p$ 's can achieve early undertaking in the same way as the tight deadline. The stochastic deadline, however, does not leave as many started tasks uncompleted as the tight, but deterministic, deadline does. Because the event of an extension allows agents to work on the task also in the last period before the natural deadline expires, more tasks get completed. The stochastic deadline improves also upon the natural deadline, precisely because it encourages early undertaking. It does so even if some tasks are left unfinished in the event that the tight deadline does not get extended. The reason is that the gain due to increased likelihood that the project gets started is greater than the loss in rate of completion when the deadline is not extended.

Proposition 6 Assume that $T \geq 3 . \pi_{T}(p, q, B)>\pi_{T}(p, B)$ whenever the stochastic deadline induces earlier start than the deterministic deadline.

Proof. See appendix

It follows immediately from proposition 6 that for some levels of distraction, $p$, and for a given payment to the agent, the principal can achieve higher profits, compared to granting maximal time, if he is able to commit to a stochastic deadline which induces the agent to get started early. This result rests on the assumption that the payment cannot be made contingent on the date of completion and that payment to the agent is determined by some unmodelled ex-post bargaining. In what follows, we show by means of two examples that stochastic deadlines can be more profitable than deterministic ones, also under less restrictive assumptions regarding payments. In particular, we explore the principal's incentive to impose a stochastic deadline when (i) the principal can also choose the payment to the agent and (ii) when a fraction of the fixed $B$ can be paid upon completion, rather than at the deadline.

The first example, depicted in Figure 8, explores the possibility that principals set $B$ and $q$ jointly to maximize expected profits. Panel panel i) shows, for each $q \in[0,1]$, the minimum payment that gives the agent the incentives to start $k(k=2,3,4)$ periods before a deadline 
set at 4 , i.e; $s_{k}^{S}(p, q)$. The thick line corresponds to $k=4$, the dashed line to $k=3$ and the dotted line to $k=2$. Panel $i i)$ depicts the principal's profits under those payments. The highest profit is reached at $q=q_{1}^{*}$ : the principal chooses $q$ so that $s_{4}^{S}(p, q)=s_{3}^{S}(p, q)$. For smaller $q^{\prime} s$ it is easier to induce the agent to start 4 periods before the deadline than 3 periods before, the opposite is true for larger $q^{\prime} s$. To induce the agent to start 2 periods ahead of the deadline is only worthwhile when $q$ is close to 1 .
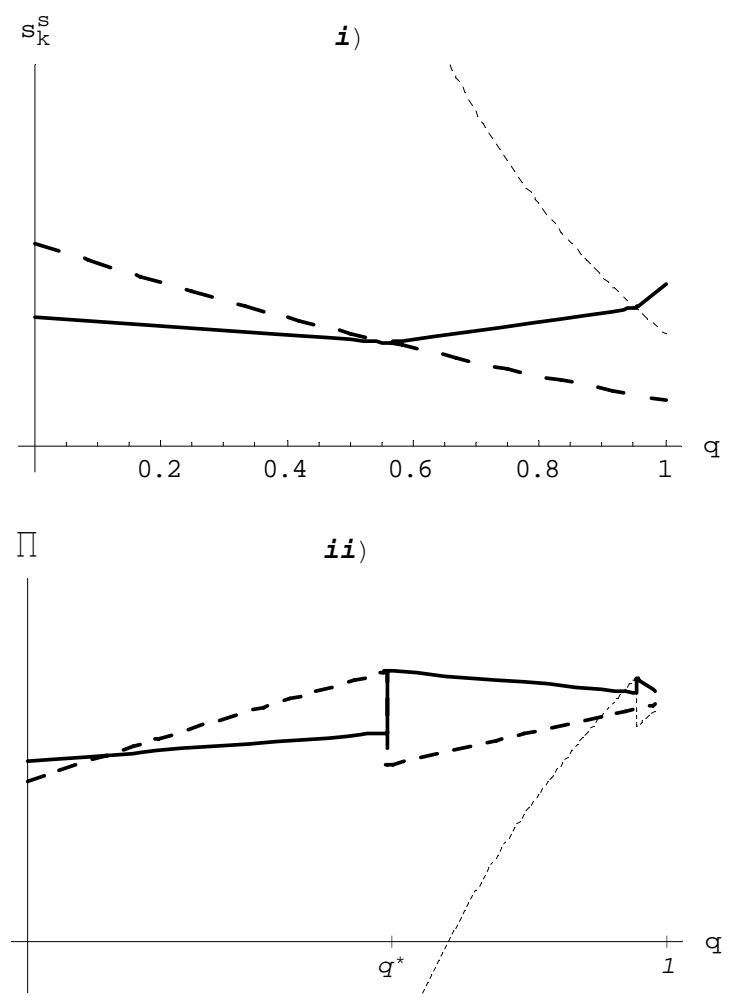

Figure 8: i) Payments $s_{k}^{s}(p, q), k=2,3,4 \quad(\mathrm{p}=0.35)$. ii) Corresponding profits with a stochastic deadline at $T=4$.

Figure 9 shows that a stochastic deadline can be superior to a deterministic deadline also when advance payments are allowed. We assume that the reward $B$ is fixed, but as an alternative to imposing a stochastic deadline at $T$, we allow the principal to pay $\alpha B(\alpha \in[0,1])$ upon completion. The remainder $(1-\alpha) B$ is paid at the deadline $T$. We have chosen the parameters such that the agent would start the second and third period $(k=2,3)$ but not the first $(k=4)$ if the deadline were deterministic at 4 (i.e; $q=1$ ). In the horizontal axis we have both $q$ and $\alpha$. The thick line shows how profits change with $q$ (with $\alpha=0$, i.e, no advance payments). The maximum is reached at $q=q^{*}$. For such probability of extension, the agent is willing to start the first period $(k=4)$. The thin line depicts profits as a function 
of the share of advance payments, $\alpha$ (with $q=1$, i.e, deterministic deadline). The maximum is reached at $\alpha=\alpha^{*}$. For such share, agents start also the first period. As the fraction offered in advance payment increases beyond $\alpha^{*}$, the present discounted value of profits decline.

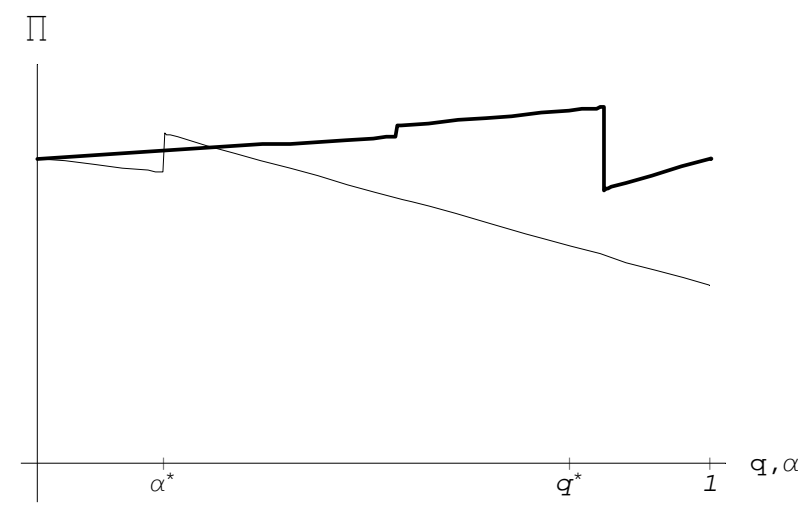

Figure 9: Stochastic deadlines vs deterministic deadlines with advance payments, $\mathrm{T}=4$, $\mathrm{p}=0.2$.

\section{Conclusion}

We have studied the timing of effort and the probability of completing a task which is carried out by time-consistent agents who are occasionally distracted and prevented from working. Our first result is that, given the length of the deadline, agents who are more frequently distracted may complete with higher probability than a agents who are less likely to be distracted. The intuition for this result is that the former start working earlier for precautionary motives.

Furthermore, our analysis has shown that although stochastic deadlines are by construction a convex combination of deterministic deadlines, the resulting probability of completion is not. The probability of completion with stochastic deadlines dominates both "tight" and "natural" deadlines whenever the threat of a tighter deadline encourages the agent to get started earlier. In the light of this result, there is no need to invoke time-inconsistency to understand why setters of many real world deadlines set deadlines tighter than those imposed by nature and that they occasionally extend the deadlines they originally set.

Understanding how deadlines and distractions affect how agents trade off the probability of completing against the desire to postpone effort is of interest to setters of deadlines, be it contractors, employers or grant funders who are concerned with timely completion of tasks. our results indicate that it may be beneficial for principals to invest in structures and routines that are reasonably, but not completely, flexible such that commitment to tight deadlines is imperfect. 
Throughout this paper we have assumed that there is no cost in spreading the two periods needed to complete the task. An extension incorporating the possibility of depreciation of the work carried out the first period is equivalent to introducing a deadline within the original deadline. We conjecture that the qualitative results will remain unchanged and that for high enough payments, the agent may be willing to re-start in case the initial investment is lost. Another route for future research is to investigate optimal deadline regimes when principals have an imperfect knowledge of the agents' probability of distraction. These and other extensions are left for future research.

\section{References}

ASA (2002): "HIPAA Extension; Privacy Rule Changes," American Society of Anesthesiologists Newsletter, vol 66, no 5.

Boice, R. (1989): "Procrastination, Busyness and Bingeing," Behaviour Research and Therapy, 27 (6), 605-611.

EC (2003): "European Commission: Important Legal Notice 04.04.2003," European Commission.

Fischer, C. (2001): "Read This Paper Later: Procrastination with Time-Consistent Preferences," Journal of Economic Behaviour and Organization, vol 46(3), 249-269.

Grossman, S. J., and O. D. Hart (1986): "The Costs and Benefits of Ownership: A Theory of Vertical and Lateral Integration," Journal of Political Economy, 94(4), 691-719.

O’Donoghue, T., and M. Rabin (1999): "Incentives for Procrastinators," The Quarterly Journal of Economics, CXIV, 769-816.

Solomon, L. J., and E. D. Rothblum (1984): "Academic Procrastination: Frequency and Cognitive-Behavioral Correlates," Journal of Counseling Psychology, 31(4), 503-509.

Toxvaerd, F. (2006): "Time of the Essence," Journal of Economic Theory, 129(1), 252-272.

(2007): "A Theory of Optimal Deadlines," Journal of Economic Dynamics and Control, 31, 493-513.

\section{Appendix:}

Proof of Proposition 1. We show that there exist no $k>1$ such that $T-k$ is the last period the agent wants to complete. First, assume that this is not the case and that $T-k$ is 
the last period the agent wants to complete. Then

$$
F_{k}(p, B)=\delta^{k} B+\sum_{i=1}^{k-1} \delta^{i}(1-p) \underline{u} \geq \underline{u}+\sum_{i=1}^{k-1} \delta^{i}(1-p) \underline{u}
$$

which can written as

$$
B \geq \frac{\underline{u}}{\delta^{k}}
$$

If (11) holds for some $\bar{k} \geq 2$, then it also holds for any $k=1,2, \ldots, \bar{k}-1$. Using this, we now derive the complete condition when the deadline is $k$ periods ahead, $c_{k}(p)$. Assume first that the agent wants to complete in $k-1, \ldots, 1$ periods ahead of the deadline, i.e. $B \geq c_{k-i}(p)$ for $i=1,2, \ldots, k-1$. The agent will complete $k$ periods ahead of the deadline, given that he will finish at the first opportunity thereafter, when

$$
\begin{aligned}
F_{k}(p, B) & \geq \underline{u}+\sum_{i=1}^{k-1} \delta^{i} p^{i-1}(1-p) F_{k-i}(p, B) \\
& =\underline{u}+\delta G_{k-1}(p) \underline{u}+\delta^{k} B\left(1-p^{k-1}\right),
\end{aligned}
$$

where

$$
G_{t}(p) \underline{u}=\sum_{i=0}^{t-1} \delta^{i} p^{i}(1-p) \sum_{z=1}^{t-i-1} \delta^{z}(1-p) \underline{u}
$$

is the leisure utility enjoyed after completion. Solving for $B$, the agent will complete whenever

$$
B \geq \frac{\underline{u}}{\delta^{k} p^{k-1}}\left(1+\delta G_{k-1}(p)-\sum_{i=1}^{k-1} \delta^{i}(1-p)\right)=c_{k}(p) .
$$

Expanding the terms in the RHS of (14), we obtain

$$
c_{k}(p)=\frac{\underline{u}}{\delta^{k} p^{k-1}}\left(1-(1-p) \frac{\delta p-(\delta p)^{k}}{p(1-\delta p)}\right) \text { for } k=1,2 \ldots
$$

It is easy to see that $c_{k}^{\prime}(p)<0, \lim _{p \rightarrow 0} c_{k}(p)=\infty$ for all $k=2,3 \ldots$. Note that

$$
c_{k}(p)=\left(c_{k-1}(p)-(1-p) \underline{u}\right) / \delta p>c_{k-1}(p) .
$$

This completes the proof.

Proof of Lemma 1. As a preliminary step we introduce some notation. $I_{k}^{i} \in\{0,1\}$ is an indicator of the agent's decision $k$ periods before the deadline (at $T-k$ ), given that he has worked $i$ times before $(i=0,1)$. It takes value 1 when the agent plans to work at $T-k$ and 0 when he does not plan to work. $V_{k}\left(i, p, B, \mathbf{I}_{k}\right)$ denotes the agent's present discounted expected 
payoff prior to the realization of the uncertainty at $T-k$, given the number of periods in which the agent previously worked, $i$, the probability of being distracted, $p$, the payoff, $B$, and his planned behavior from period $T-k$ onwards, $\mathbf{I}_{k}=\left(I_{k}, I_{k-1}, . ., I_{1}\right)$, where $I_{t}=\left\{I_{t}^{0}, I_{t}^{1}\right\}$. $V_{k}\left(i, p, B, \mathbf{I}_{k}\right)$ can be written as a convex combination of the continuation payoff in case the opportunity cost of time at $T-k$ is high and the continuation payoff in case it is low:

$$
V_{k}\left(i, p, B, \mathbf{I}_{k}\right)=(1-p)\left(\left(1-I_{k}^{i}\right) \underline{u}+\delta V_{k-1}\left(i+I_{k}^{i}, p, B, \mathbf{I}_{k-1}\right)\right)+p \delta V_{k-1}\left(i, p, B, \mathbf{I}_{k-1}\right) .
$$

The solution to the agent's decision problem at each time $T-k$, is the sequence $\mathbf{I}_{k}=\left\{I_{t}\right\}_{t=1}^{k}$ that maximizes (16). Since the agent is time consistent, $\mathbf{I}_{k}^{*}=\left(I_{k}^{*}, \mathbf{I}_{k-1}^{*}\right)$ for all $k=0,1, \ldots$

Assume that $s_{k}(p)>B \geq \max \left[s_{k-1}(p), s_{k+1}(p)\right]$, then the three following inequalities hold simultaneously:

$$
\begin{aligned}
0+\delta V_{k}\left(1, p, B, \mathbf{I}_{k}^{*}\right) & \geq \underline{u}+\delta V_{k}\left(0, p, B, \mathbf{I}_{k}^{*}\right), \\
0+\delta V_{k-1}\left(1, p, B, \mathbf{I}_{k-1}^{*}\right) & <\underline{u}+\delta V_{k-1}\left(0, p, B, \mathbf{I}_{k-1}^{*}\right), \\
0+\delta V_{k-2}\left(1, p, B, \mathbf{I}_{k-2}^{*}\right) & \geq \underline{u}+\delta V_{k-2}\left(0, p, B, \mathbf{I}_{k-2}^{*}\right) .
\end{aligned}
$$

Subtracting (18) from (17) we obtain that

$$
V_{k}\left(1, p, B, \mathbf{I}_{k}^{*}\right)-V_{k-1}\left(1, p, B, \mathbf{I}_{k-1}^{*}\right)>V_{k}\left(0, p, B, \mathbf{I}_{k}^{*}\right)-V_{k-1}\left(0, p, B, \mathbf{I}_{k-1}^{*}\right) .
$$

By assumption the agent does not want to start $k$ periods ahead of the deadline. Thus

$$
V_{k}\left(0, p, B, \mathbf{I}_{k}^{*}\right)>(1-p) \delta V_{k-1}\left(1, p, B, \mathbf{I}_{k-1}^{*}\right)+p \delta V_{k-1}\left(0, p, B, \mathbf{I}_{k-1}^{*}\right)
$$

and

$$
V_{k-1}\left(0, p, B, \mathbf{I}_{k-1}^{*}\right)=(1-p) \delta V_{k-2}\left(1, p, B, \mathbf{I}_{k-2}^{*}\right)+\delta p V_{k-2}\left(0, p, B, \mathbf{I}_{k-2}^{*}\right),
$$

since by assumption he wants to start $k-1$ periods ahead of the deadline. Substituting for $V_{k}\left(0, p, B, \mathbf{I}_{k}^{*}\right)$ in (20) by the RHS of $(21)$ and for $V_{k-1}\left(0, p, B, \mathbf{I}_{k-1}^{*}\right)$ by $(22)$, the inequality in (20) still holds,

$$
\begin{aligned}
& V_{k}\left(1, p, B, \mathbf{I}_{k}^{*}\right)-V_{k-1}\left(1, p, B, \mathbf{I}_{k-1}^{*}\right)>\delta\left(V_{k-1}\left(1, p, B, \mathbf{I}_{k-1}^{*}\right)-V_{k-2}\left(1, p, B, \mathbf{I}_{k-2}^{*}\right)\right) \\
& +p \delta\left(V_{k-2}\left(1, p, B, \mathbf{I}_{k-2}^{*}\right)-V_{k-2}\left(0, p, B, \mathbf{I}_{k-2}^{*}\right)\right)-p \delta\left(V_{k-1}\left(1, p, B, \mathbf{I}_{k-1}^{*}\right)-V_{k-1}\left(0, p, B, \mathbf{I}_{k-1}^{*}\right)\right) .
\end{aligned}
$$

a) Assume first that the agent is willing to complete $k$ periods ahead of the deadline. Since $c_{k}(p)>c_{k-i}(p)$ for all $i>0$, the agent also wants to finish in all consecutive occasions and

$$
\begin{aligned}
V_{k}\left(1, p, B, \mathbf{I}_{k}^{*}\right) & =(1-p) F_{k}(p, B)+\delta p V_{k-1}\left(1, p, B, \mathbf{I}_{k-1}^{*}\right) \\
& =\sum_{i=0}^{k-1} \delta^{i} p^{i}(1-p) F_{k-i}(p, B)
\end{aligned}
$$


Substituting for $V_{k}\left(1, p, B, \mathbf{I}_{k}^{*}\right)$ and $V_{k-1}\left(1, p, B, \mathbf{I}_{k-1}^{*}\right)$ in (23) by (24) and rearranging we get:

$$
\begin{aligned}
& (1-p)\left(F_{k}(p, B)-F_{k-1}(p)\right)+(\delta p-\delta)\left(V_{k-1}\left(1, p, B, \mathbf{I}_{k-1}^{*}\right)-V_{k-2}\left(1, p, B, \mathbf{I}_{k-2}^{*}\right)\right)> \\
& \delta p\left(V_{k-2}\left(1, p, B, \mathbf{I}_{k-2}^{*}\right)-V_{k-2}\left(0, p, B, \mathbf{I}_{k-2}^{*}\right)-\delta p\left(V_{k-1}\left(1, p, B, \mathbf{I}_{k-1}^{*}\right)-V_{k-1}\left(0, p, B, \mathbf{I}_{k-1}^{*}\right)\right)\right) .
\end{aligned}
$$

The RHS of (25) is positive, since $V_{k-1}\left(1, p, B, \mathbf{I}_{k-1}^{*}\right)-V_{k-1}\left(0, p, B, \mathbf{I}_{k-1}^{*}\right)<\underline{u} / \delta$, and $V_{k-2}\left(1, p, B, \mathbf{I}_{k-2}^{*}\right)-V_{k-2}\left(0, p, B, \mathbf{I}_{k-2}^{*}\right) \geq \underline{u} / \delta$. Substituting $F_{k}(p, B)$ by $(10)$ and $V_{k-1}\left(1, p, B, \mathbf{I}_{k-1}^{*}\right)$ by $(24)$ in the LHS of $(25)$ we obtain

$$
\begin{aligned}
& (1-p)\left(F_{k}(p, B)-F_{k-1}(p, B)\right)+(\delta p-\delta)\left(V_{k-1}\left(1, p, B, \mathbf{I}_{k-1}^{*}\right)-V_{k-2}\left(1, p, B, \mathbf{I}_{k-2}^{*}\right)\right)= \\
& (1-p) p^{k-2} \delta^{k-1}((1-p) \underline{u}-B(1-p \delta)),
\end{aligned}
$$

which can only be positive if

$$
B \leq \underline{u} \frac{1-p}{1-p \delta}<\frac{u}{\delta}
$$

Since $\delta B>\underline{u}$, the LHS of (25) cannot be positive. Hence, (17), (18) and (19) cannot hold simultaneously if the agent wants to complete the task at $T-k$.

b) Assume now that the agent does not want to complete at $T-k$. We can now write $V_{k}\left(1, p, B, \mathbf{I}_{k}^{*}\right)$ as

$$
V_{k}\left(1, p, B, \mathbf{I}_{k}^{*}\right)=(1-p) \underline{u}+\delta V_{k-1}\left(1, p, B, \mathbf{I}_{k-1}^{*}\right) .
$$

If $V_{k-1}\left(1, p, B, \mathbf{I}_{k-1}^{*}\right)$ is subtracted from both sides, this allows us to write the inequality in (20) as

$$
\begin{aligned}
& (1-p) \underline{u}-(1-\delta) V_{k-1}\left(1, p, B, \mathbf{I}_{k-1}^{*}\right)> \\
& (1-p) \underline{u}+\delta V_{k-1}\left(0, p, B, \mathbf{I}_{k-1}^{*}\right)-V_{k-1}\left(0, p, B, \mathbf{I}_{k-1}^{*}\right),
\end{aligned}
$$

where we have used the assumption that the agent does not want to start $k$ periods ahead of the deadline i.e., $V_{k}\left(0, p, B, \mathbf{I}_{k}^{*}\right)=(1-p) \underline{u}+\delta V_{k-1}\left(0, p, B, \mathbf{I}_{k-1}^{*}\right)$. The inequality in (26) can be re-written as

$$
\begin{aligned}
& \delta\left(V_{k-1}\left(1, p, B, \mathbf{I}_{k-1}^{*}\right)-V_{k-1}\left(0, p, B, \mathbf{I}_{k-1}^{*}\right)\right)> \\
& V_{k-1}\left(1, p, B, \mathbf{I}_{k-1}^{*}\right)-V_{k-1}\left(0, p, B, \mathbf{I}_{k-1}^{*}\right),
\end{aligned}
$$

which cannot hold for $\delta<1$ and equations (17), (18) and (19) cannot hold simultaneously. This completes the proof.

Proof of Lemma 2. Assume that $T-k$ is the last period the agent wants to start, i.e. $k=$ $\underline{k}^{s}$ and that $k^{c}<\underline{k}^{s}-1$. The agent starts at $T-k$, whenever

$$
\delta V_{k-1}\left(1, p, B, \mathbf{I}_{k-1}^{*}\right) \geq \underline{u}+\delta V_{k-1}\left(0, p, B, \mathbf{I}_{k-1}^{*}\right),
$$


which can be written as

$$
\sum_{i=1}^{k-1-k^{c}} \delta^{i}(1-p) \underline{u}+\delta^{k-k^{c}} \sum_{j=0}^{k^{c}-1} \delta^{j} p^{j}(1-p) F_{k^{c}-j}(p, B) \geq \underline{u}+\sum_{i=1}^{k-1} \delta^{i}(1-p) \underline{u} .
$$

Adding $\delta^{k-1-k^{c}} \underline{u}$ on both sides of (27) and simplifying we get

$$
\delta^{k-1-k^{c}}\left(\underline{u}+\sum_{j=1}^{k^{c}} \delta^{j} p^{j-1}(1-p) F_{k^{c}+1-j}(p, B)-\sum_{i=1}^{k^{c}} \delta^{i}(1-p) \underline{u}\right) \geq \underline{u}\left(1+\delta^{k-1-k^{c}}\right),
$$

which using the LHS of (12) and (14) can be written as

$$
\delta^{k} p^{k^{c}}\left(c_{k^{c}+1}(p)-B\right) \geq \underline{u}\left(1+\delta^{k-1-k^{c}}\right)-\delta^{k} B .
$$

Since $k=\underline{k}^{s}(p, B)$, the agent does not want to start at $T-k-1$, and because $T-k^{c}$ is the first period the agent wants to complete, it is the case that

$$
\delta^{k-1} p^{k^{c}}\left(c_{k^{c}+1}(p)-B\right)<\underline{u}\left(1+\delta^{k-2-k^{c}}\right)-\delta^{k-1} B .
$$

A necessary condition for(28) and (29) to hold simultaneously is that

$$
\underline{u}\left(1+\delta^{k-1-k^{c}}\right)-\delta^{k} B<\delta\left(\underline{u}\left(1+\delta^{k-2-k^{c}}\right)-\delta^{k-1} B\right),
$$

which is satisfied whenever

$$
1<\delta
$$

This cannot hold, and hence $k^{c}+1 \geq \underline{k}^{s}$.

Proof of Proposition 2. Consider the agent's decision to start at $T-k$. If he starts, he plans to complete at first when $m \in\{k-1, k-2, . ., 1\}$ periods remain. If he does not start, he plans to start at the latest when $n \in\{k, k-1, . .2\}$ periods remain. Assume that $m \geq n-1$. Let $W_{k}^{s}(p, B, m)$ be the present value at $T-k$ of $s$ tarting and $W_{k}^{w}(p, B, m, n)$ the value of postponing start (waiting) given that the opportunity cost at $k$ is low, the first completion date is $T-m$ and last starting date $T-n$. The agent starts if $B$ is such that

$$
\begin{aligned}
W_{k}^{s}(p, B, m) & =L_{k}^{s}(p, m) \underline{u}+\pi_{k}^{s}(p, m) \delta^{k} B \geq \\
L_{k}^{w}(p, m, n) \underline{u}+\pi_{k}^{w}(p, m, n) \delta^{k} B & =W_{k}^{w}(p, B, m, n)
\end{aligned}
$$

where

$$
\pi_{k}^{s}(p, m)=1-p^{m}
$$

is the probability that the agent completes a task if he starts it at $T-k$ and plans to complete at $T-m$ at the earliest,

$$
L_{k}^{s}(p, m) \underline{u}=\sum_{i=1}^{k-m-1} \delta^{i}(1-p) \underline{u}+\delta^{k-m} \sum_{j=0}^{m-1} \delta^{j} p^{j}(1-p) \sum_{z=1}^{m-j-1} \delta^{z}(1-p) \underline{u}
$$


is the present value of leisure (ignoring $\bar{u}$ realizations). Part of this leisure is enjoyed before completion, and part is enjoyed after completion if the agent completes ahead of the deadline

$$
\pi_{k}^{w}(p, m, n)=\sum_{i=1}^{k-n} p^{i-1}(1-p) \pi_{k-i}^{s}(p, \min \{m, k-i-1\})
$$

is probability that the agent ever completes the project if he postpones start, and

$$
L_{k}^{w}(p, m, n) \underline{u}=\underline{u}+\sum_{i=1}^{k-n} p^{i-1}(1-p) \delta^{i} L_{k-i}^{s}(p, \min \{m, k-i-1\}) \underline{u}+p^{k-n} \delta^{k-n} \sum_{i=1}^{n-1} \delta^{i}(1-p) \underline{u}
$$

is the present expected value of leisure utility. Note that both $W_{k}^{s}(p, B, m)$ and $W_{k}^{w}(p, B, m, n)$ are linearly increasing in $B$. Moreover,

$$
\pi_{k}^{s}(p, m)>\pi_{k}^{w}(p, m, n) \text { and } L_{k}^{s}(p, m) \underline{u}<L_{k}^{w}(p, m, n) \underline{u} .
$$

Hence, there exist a unique $B=b_{k}(p, m, n)$, which makes $W_{k}^{s}(p, B, m)$ and $W_{k}^{w}(p, B, m, n)$ equal:

$$
b_{k}(p, m, n)=\frac{\underline{u}}{\delta^{k}} \frac{L_{k}^{w}(p, m, n)-L_{k}^{s}(p, m)}{\Delta \pi_{k}(p, m, n)} .
$$

The numerator is the increase in leisure utility derived from postponement of start and the denominator is the increase in the probability of completion due to starting at $T-k$ rather than waiting:

$$
\begin{aligned}
\Delta \pi_{k}(p, m, n) & =\pi_{k}^{s}(p, m)-\pi_{k}^{w}(p, m, n) \\
& =p^{k-m}-p^{k-1}+(1-p)(m-n+1) p^{k-2} .
\end{aligned}
$$

Note first that $b_{2}(p, 1,2)=s_{2}(p)$ (eq. (3)). Furthermore

1. if $B=b_{k}(p, m, n)$ and $m \neq \min \left\{k^{c}(p, B), k-1\right\}=m_{k}^{*}$ then $B>b_{k}\left(p, m_{k}^{*}, n\right)$. If the agent is indifferent between starting at $T-k$ and postponing when he is completing at suboptimal dates, i.e. then he must prefer to start when his completion strategy is optimal,

2. if $B=b_{k}\left(p, m_{k}^{*}, n\right)$ and $n \neq \underline{k}^{s}(p, B)=n^{*}$ then $b_{k}\left(p, m_{k}^{*}, n^{*}\right)>B$ : If the agent is indifferent between starting and postponing start, given that he will complete at optimal dates, but that he would start at suboptimal dates, $n \neq \underline{k}^{s}(p, B)$, then he must strictly prefer to postpone, given that he would both start and complete at optimal dates in the future.

It follows 1, 2 and Lemma 2 that the agent will start $k$ periods ahead of the deadline if

$$
B \geq \max _{n \in\{k, k-1, . .2\}} \min _{m \in\{k-1, . . n-1\}} b_{k}(p, m, n)=s_{k}(p) .
$$


For $k \geq 3, s_{k}(p)$ is U-shaped with $\lim _{p \rightarrow 0} s_{k}(p)=\infty$ and $\lim _{p \rightarrow 1} s_{k}(p)=\infty$. This follows from the fact that $b_{k}(p, m, n)$ is U-shaped with $\lim _{p \rightarrow 0} b_{k}(p, m, n)=\infty$ for all $n<k$ and $\lim _{p \rightarrow 1} b_{k}(p, m, n)=\infty$ for all $n \leq k$.

3. $s_{k}(p)>s_{k-1}(p)$ for all $p$ and all $k$ if $\delta \leq 1 / 2$. This follows from the fact that $s_{3}(p)>s_{2}(p)$ for all $p$ when $\delta \leq 1 / 2$ and from corollary $1, \mathrm{i})$.

4. For $k, n \geq 3, b_{k}(p, m, n)$ can be written as

$$
\begin{aligned}
b_{k}(p, m, n)= & \frac{b_{k-1}(p, m-1, n-1)}{\delta} \frac{\Delta \pi_{k-1}(p, m-1, n-1)}{\Delta \pi_{k}(p, m, n)}+ \\
& \frac{\underline{u}(1-p)}{\delta} \frac{(p-(1-p)(m-n+1)) p^{k-3}}{\Delta \pi_{k}(p, m, n)}
\end{aligned}
$$

since

$$
\begin{aligned}
L_{k}^{s}(p, m) \underline{u}= & L_{k-1}^{s}(p, m-1) \underline{u}+\pi_{k-1}^{s}(p, m-1)(1-p) \delta^{k-1} \underline{u}, \\
L_{k}^{w}(p, m, n) \underline{u}= & L_{k-1}^{w}(p, m-1, n-1) \underline{u}+ \\
& \pi_{k-1}^{w}(p, m-1, n-1)(1-p) \delta^{k-1} \underline{u}+p^{k-n}(1-p) \delta^{k-1} \underline{u},
\end{aligned}
$$

and

$$
p^{k-n}-\Delta \pi_{k-1}(p, m-1, n-1)=(p-(1-p)(m-n+1)) p^{k-3} .
$$

Corollary 1 implies that if $s_{k}(p)<s_{k-1}(p)$, then $s_{k}(p)=b_{k}(p, k-1, k)$ and $s_{k-1}(p)=$ $b_{k-1}(p, k-2, k-1)$. Substituting $m=k$, and $n=m-1$ in (34),

$$
b_{k}(p, k-1, k)=\frac{b_{k-1}(p, k-2, k-1)}{\delta} \frac{1-p^{k-2}}{1-p^{k-1}} .
$$

$b_{k}(p, k-1, k)<b_{k-1}(p, k-2, k-1)$ whenever

$$
\delta>\frac{1-p^{k-2}}{1-p^{k-1}}=\frac{\sum_{i=0}^{k-3} p^{i}}{\sum_{i=0}^{k-2} p^{i}} .
$$

It is easy to see that (38) holds for large enough $p / s$, provided that

$$
\delta>\frac{k-2}{k-1}=\frac{\sum_{i=0}^{k-3} 1^{i}}{\sum_{i=0}^{k-2} 1^{i}} .
$$


Proof of Proposition 3. i) and ii) follow directly from (5). iii) It follows from (5), that, given $B, \pi_{T}(p, B)$ is continuously decreasing in $p$ whenever changes in $p$ do not involve changes in the agent's starting and completing decisions.

Assume $B>s_{k}(p)$ for some $k \in\{3, . . T\}$ and some $p$ and let $\underline{p_{k}}(B)$ be the smallest of the two solutions in $(0,1)$ to

$$
B=s_{k}(p) \text {. }
$$

Since $s_{k}(p)$ is $\mathrm{U}$-shaped, we can find an arbitrarily small $\epsilon>0$ such that $B<s_{k}\left(\underline{p_{k}}(B)-\epsilon\right)$. The set of dates in which the agent is willing to start is larger for $p=p_{k}(B)$ than for $p=\underline{p_{k}}(B)-\epsilon$, with all other decisions remaining unchanged. It follows from $\overline{(5)}$ that $\pi_{T}\left(\underline{p_{k}}(B) \overline{-} \epsilon, B\right)<$ $\pi_{T}\left(\underline{p_{k}}(B), B\right)$.

Proof of Proposition 4. Let $c_{k}^{S}(p, q)$ denote the complete condition when the stochastic deadline is $k$ periods away. Assume that $B>c_{k-i}^{S}(p, q)$ for $i=1,2 . . k-1$. The agent will complete $k$ periods ahead of the deadline when

$$
\begin{aligned}
F_{k}(p, B) \geq & \underline{u}+L_{k}^{s}(p, k-1) \underline{u}+p^{k-2}(1-p)(1-q) \delta^{k-1} \underline{u}+ \\
& \left(1-p^{k-2}+p^{k-2}(1-p) q\right) \delta^{k} B .
\end{aligned}
$$

With a deterministic deadline the agent would have finished in the last period had he experienced $k-2$ periods of high opportunity cost. With the stochastic deadline he cannot work in the last period with probability $(1-q)$. The above expression can be re-arranged to obtain the following complete condition

$$
B \geq \frac{p c_{k}(p)}{1-(1-p) q}+\frac{(1-q)(1-p) \underline{u}}{\delta(1-(1-p) q)}=c_{k}^{S}(p, q) .
$$

Note that $c_{k}^{S}(p, 1)=c_{k}(p)$. It follows from $(15)$ that $c_{k}^{S}(p, 0)=c_{k-1}(p) / \delta$. Moreover $c_{k}^{S}(p, q)>$ $c_{k-1}^{S}(p, q)$ and $\partial c_{k}^{S}(p, q) / \partial q>0$. The agent is more willing to complete when the deadline is stochastic.

Proof of Lemma 3. Assume that the deadline is stochastic at $T$ and that the agent considers starting $k \geq 3$ periods before the deadline. If he starts, he plans to complete at first when $m \in\{k-1, k-2, . ., 1\}$ periods remain. If he does not start, he plans to start at the latest when $n \in\{k, k-1, . .2\}$ periods remain. The agent is willing to start at $T-k$ if

$$
\begin{aligned}
& q W_{k}^{s}(p, B, m)+(1-q)\left(W_{k-1}^{s}(p, \delta B, m-1)+(1-p) \delta^{k-1} \underline{u}\right) \\
\geq & q W_{k}^{w}(p, B, m, n)+(1-q)\left(W_{k-1}^{W}(p, \delta B, m-1, n-1)+(1-p) \delta^{k-1} \underline{u}\right)
\end{aligned}
$$

The agent will start when $B \geq b_{k}^{S}(p, m, n, q)$

$$
\begin{aligned}
B \geq & b_{k}^{S}(p, m, n, q)=q \frac{\underline{u}}{\delta^{k}} \frac{L_{k}^{w}(p, m, n)-L_{k}^{s}(p, m)}{q \Delta \pi_{k}(p, m, n)+(1-q) \Delta \pi_{k-1}(p, m-1, n-1)}+ \\
& (1-q) \frac{\underline{u}}{\delta^{k}} \frac{L_{k-1}^{w}(p, m-1, n-1)-L_{k-1}^{s}(p, m-1)}{q \Delta \pi_{k}(p, m, n)+(1-q) \Delta \pi_{k-1}(p, m-1, n-1)} .
\end{aligned}
$$


Note that

$$
\frac{\partial b_{k}^{s}(p, m, n, q)}{\partial q}=\frac{(1-p)\left(b_{k}^{s}(p, m, n, q)-\underline{u} / \delta\right)((1-p)(m-n+1)-p) p^{k-3}}{q \Delta \pi_{k}(p, m, n)+(1-q) \Delta \pi_{k-1}(p, m-1, n-1)}
$$

where we have used (35), (36), and (37). Since $b_{k}^{s}(p, m, n, q)>\underline{u} / \delta$, the derivative is positive (negative) whenever

$$
p \lesseqgtr \frac{m-n+1}{m-n+2}=p(m, n) .
$$

Note that since $m \geq n-1, p(m, n) \geq 0$.

Let $m_{k}(p, q)$ and $n_{k}(p, q)$ solve

$$
\max _{n \in\{k, k-1, . .2\}} \min _{m \in\{k-1, . . . n-1\}} b_{k}(p, m, n, q) .
$$

The agent will start $k$ periods ahead of the deadline if

$$
B \geq b_{k}\left(p, m_{k}(p, q), m_{k}(p, q), q\right)=s_{k}^{s}(p, q) .
$$

Proof of proposition 5. If $k$ is the last period the agent wants to start, $s_{k}(p, q)=$ $b_{k}(p, k-1, k, q)$, and decreases in $q$ lead to increases in $s_{k}(p, q)$. Whenever $m(q) \geq n(q)-1$, decreases in $q$ lead to decreases in $s_{k}$, provided $p$ is small enough.

Proof of Proposition 6. Assume the first period the agent is willing to start with a deterministic deadline $T$ periods away is when $T-1$ periods remain, i.e. $s_{T}(p)>B>s_{T-1}(p)$. The total probability of completion is

$$
\pi_{T}(p, B)=\pi_{T-1}(p, B)
$$

If the stochastic deadline motivates early undertaking, i.e. $B>s_{T}^{s}(p, q)$ the total probability of completing, $\pi_{T}^{S}(p, B, q)$, is

$$
\pi_{T}^{S}(p, B, q)=\pi_{T-1}(p, B)+\gamma q(1-p)>\pi_{T-1}(p, B),
$$

where $\gamma \in(0,1)$ is the probability that the agent has started, but not completed by $t=T-1$. 